\title{
ADSORÇÃO DE COBALTO UTILIZANDO CASCA DE ARROZ E INFLUENNCIA DO TRATAMENTO SUPERCRÍTICO
}

\author{
G. F. DÖRTZBACHER ${ }^{1}$, J. M. da CUNHA ${ }^{1}$,D. A. BERTUOL ${ }^{2}$, E. H. TANABE ${ }^{2}$ eG. L. \\ DOTTO $^{2}$
}

\begin{abstract}
${ }^{1}$ Universidade Federal de Santa Maria, Curso de Engenharia Química
${ }^{2}$ UniversidadeFederal de Santa Maria, Departamento de Engenharia Química E-mail para contato: jeaninecunha@gmail.com; guilherme_dotto@yahoo.com.br

RESUMO - O presente trabalho teve como objetivo investigar a adsorção de cobalto em casca de arroz in natura e modificada via $\mathrm{CO}_{2}$ supercrítico.No estudo cinético, utilizaram-se os modelos de pseudo-primeira ordem e pseudosegundaordem, sendo este último o mais adequado para a representação do comportamento cinético. Para o equilíbrio, utilizaram-se os modelos de Freundlich e BET, sendo o segundo o que melhor ajustou-se aos dados experimentais. A capacidade de adsorção estimada na faixa de trabalho aumentou de $13,0 \mathrm{mgg}^{-1}$ para a casca in natura para $28,8 \mathrm{mg} \mathrm{g}^{-1}$ para a modificada via $\mathrm{CO}_{2}$ supercrítico, correspondendo a um aumento favorável ao uso do tratamento.
\end{abstract}

\section{INTRODUÇÃO}

Muitos efluentes contêm o cobalto na forma de íons dissolvidos. Este se configuracomo um poluente tóxico e bioacumulável (Safa e Bhatti, 2011). A adsorção destaca-se por ser uma operação de baixo custo e acessível para a remoção de elementos em baixas concentrações. $\mathrm{O}$ carvão ativado é o mais utilizado, porém apresenta problemas de alto custo e dificuldade de reutilização (Sulaymonet al., 2009).Adsorventes alternativos têm sido estudados, comoresíduos agrícolas. Com o objetivo de reduzir a geração de resíduos em processos industriais e agrícolas, investe-se em meios de transformá-los em subprodutos de interesse comercial (Orlandoet al., 2012). A casca do arroz é o principal subproduto do processamento de arroz. O seu uso como material adsorvente tem sido discutido devido à sua altadisponibilidade, baixo custo, biodegradabilidade e possibilidade de reuso. Seu potencial uso como adsorvente é consequência de sua constituição química (Vieira et al., 2012). No entanto, sua área superficial é baixa. Uma alternativa para melhorar as propriedades da casca de arroz é o tratamento supercrítico. Neste método, a baixa viscosidade e ausência de tensão superficial contribuem para penetração do fluído na matriz sólida, aumentando os sítios de adsorção e a porosidade do material (Santos, 2011). Assim, o objetivo deste trabalho foi investigar a adsorção de cobalto em casca de arroz in natura e modificada via $\mathrm{CO}_{2}$ supercrítico.

\section{MATERIAL E MÉTODOS}

\subsection{Preparo e obtenção do adsorbato/adsorvente}


As soluções do adsorbato foram preparadas com sulfato de cobalto hepta-hidratado (99\%, Vetec, LTDA) e água deionizada.A casca de arroz foi obtida de uma indústria local. O material foi lavado com água deionizada, seco em estufa a $60{ }^{\circ} \mathrm{C}$ por 24 horas, moído em moinho de sapatas e caracterizado por peneiramento, onde partículas menores que $125 \mu \mathrm{m}$ foram utilizadas. Parte deste $(5,00 \mathrm{~g})$ recebeu o tratamento via $\mathrm{CO}_{2}$ supercrítico a $35{ }^{\circ} \mathrm{C}$ e 200 bar durante 1 hora. $\mathrm{O}$ tratamento supercrítico foi realizado em sistema montado no laboratório, contendo um reator de alta pressão, um cilindro de $\mathrm{CO}_{2}$ e uma bomba de alta pressão (500D, Teledyne). Amostras de casca de arroz tratada e in natura foram caracterizadas em relação à área superficial (Quantachrome, New Win2) e microscopia eletrônica de varredura (Jeol, JSM-6610LV).

\subsection{Ensaios de adsorção}

Os experimentos de adsorção foram efetuados em batelada, utilizando-se $50 \mathrm{~mL}$ de solução com aproximadamente $100 \mathrm{mgL}^{-1}$ de cobalto, em banho com agitação constante de $120 \mathrm{rpm}$ (Marconi LTDA). Variou-se o $\mathrm{pH}(2-6)$, o tempo (0-5 h) e a massa de adsorvente (0,05-0,5 g), de acordo com o efeito a ser estudado. Para correção dopH, utilizaram-se soluções $0,1 \mathrm{~mol} \mathrm{~L}-1$ de $\mathrm{NaOH}$ e $\mathrm{HNO}_{3}$. A concentração remanescente de cobalto na fase líquida foi determinada por absorção atômica em chama (Agilent). As capacidades de adsorção no tempo t $\left(q_{t}\right)$ e no equilíbrio $\left(q_{e}\right)$ foram obtidas pelas Equações 1 e 2, onde $C_{0}, C t$ e $C e\left(\mathrm{mg} \mathrm{L}^{-1}\right)$ são a concentrações de cobalto na fase líquida inicial, no tempo $t$ e no equilíbrio, respectivamente; $m$ (g) é a massa de adsorvente; e $V(\mathrm{~L})$ é o volume de solução.

$$
\begin{aligned}
& q_{t}=\frac{\left(C_{0}-C_{t}\right)}{m} V \\
& q_{e}=\frac{\left(C_{0}-C_{e}\right)}{m} V
\end{aligned}
$$

\subsection{Cinética de adsorção}

Os dados cinéticos foram ajustados aos modelos de pseudo-primeira e pseudo-segunda ordem (Equações3 e 4), sendo $q_{1}$ e $q_{2}\left(\mathrm{mg} \mathrm{g}^{-1}\right)$ as capacidades teóricas de adsorção; $k_{1}\left(\mathrm{~min}^{-1}\right) \mathrm{e}$ $k_{2}\left(\mathrm{~g} \mathrm{mg}^{-1} \mathrm{~min}^{-1}\right)$ as constantes cinéticas de pseudo-primeira ordem e pseudo-segunda ordem, respectivamente.

$$
\begin{array}{r}
q_{t}=q_{1}\left(1-\exp \left(-k_{1} t\right)\right) \\
q_{t}=\frac{t}{\left(1 / k_{2} q_{2}^{2}\right)+\left(t / q_{2}\right)}
\end{array}
$$

\subsection{Equilíbrio de adsorção}

Os dados experimentais obtidos no estudo do equilíbrio foram ajustados nos modelos de isotermas de Freundlich (Equação 5) e BET (Equação 6). Onde $k_{F}$ e $n_{F}$ são as constantes de Freundlich; $K_{1}, K_{2}$ e $q_{B E T}$ são as constantes do modelo BET. 


$$
\begin{aligned}
& q_{e}=k_{F} C_{e}{ }^{1 / n_{F}} \\
& q_{e}=\frac{K_{1} q_{B E T} C_{e}}{\left(1-K_{2} C_{e}\right)\left(1-K_{2} C_{e}+K_{1} C_{e}\right)}
\end{aligned}
$$

\section{RESULTADOS E DISCUSSÃO}

\subsection{Caracterização dos adsorventes}

A Figura 1 apresenta as imagens de MEV da casca de arroz in natura e tratada com fluído supercrítico.

Figura 1 - MEV da casca de arroz in natura (a) e tratada com fluído supercrítico (b).

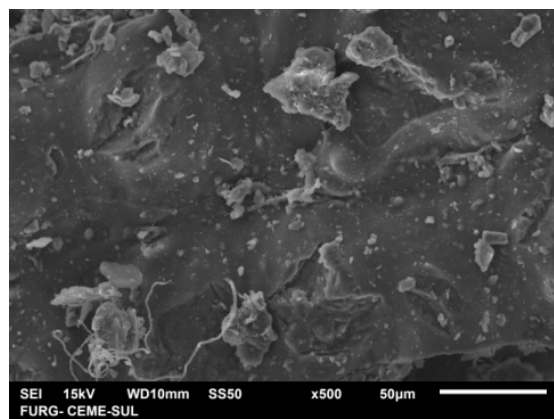

(a)

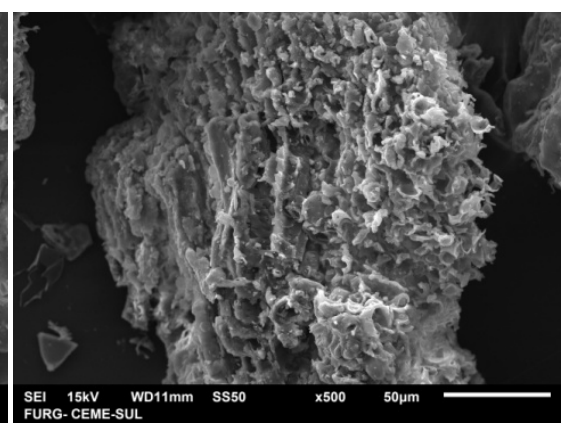

(b)

Após o tratamento, a superfície tornou-se mais rugosa, com cavidades e protuberâncias. A área superficial aumentou de $3,9 \mathrm{~m}^{2} \mathrm{~g}^{-1}$ para $15,5 \mathrm{~m}^{2} \mathrm{~g}^{-1}$.

\subsection{Efeito do pH}

A Figura 2 apresenta o efeito do $\mathrm{pH}$ na adsorção de cobalto em casca de arroz in natura e tratada via $\mathrm{CO}_{2}$ supercrítico.

Figura 2- Curva percentual de remoção em função do pH da solução.

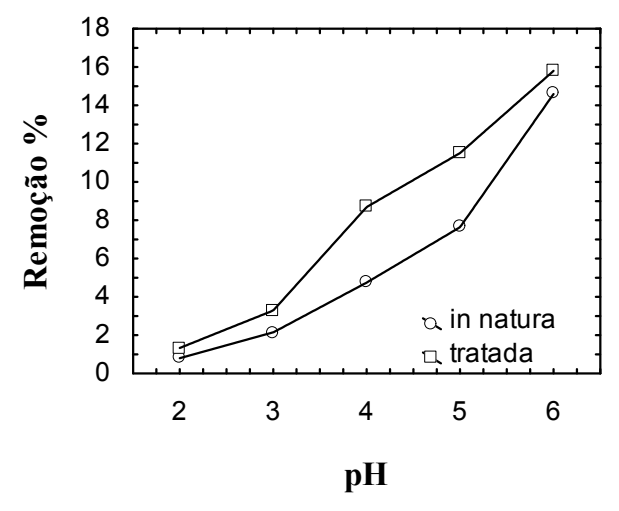


Todos ensaios foram realizados em triplicata. Experimentos preliminares indicaram que não há precipitação do adsorbato na faixa de $\mathrm{pH}$ estudada. Tanto na adsorção com casca de arroz sem tratamento quanto a tratada com $\mathrm{CO}_{2}$ supercrítico, a remoção de cobalto aumentou conforme o aumento do $\mathrm{pH}$ da solução. A influência do $\mathrm{pH}$ na adsorção pode ser explicada com base na densidade de carga superficial. Com o aumento do $\mathrm{pH}$, e consequente redução da quantidade de íons $\mathrm{H}^{+}$na superfície do adsorvente, a repulsão eletrostática entre os íons de cobalto carregados positivamente $\left(\mathrm{Co}^{+2}\right)$ e a superfície da casca de arroz diminui, o que aumenta a capacidade de adsorção. Assim, quanto maior o $\mathrm{pH}$, melhor a adsorção, desde que não ocorra a precipitação do adsorbato.

\subsection{Estudo cinético}

A Figura3 representa os dados experimentais obtidos no estudo cinético.

Figura 3 - Curva cinética de adsorção.
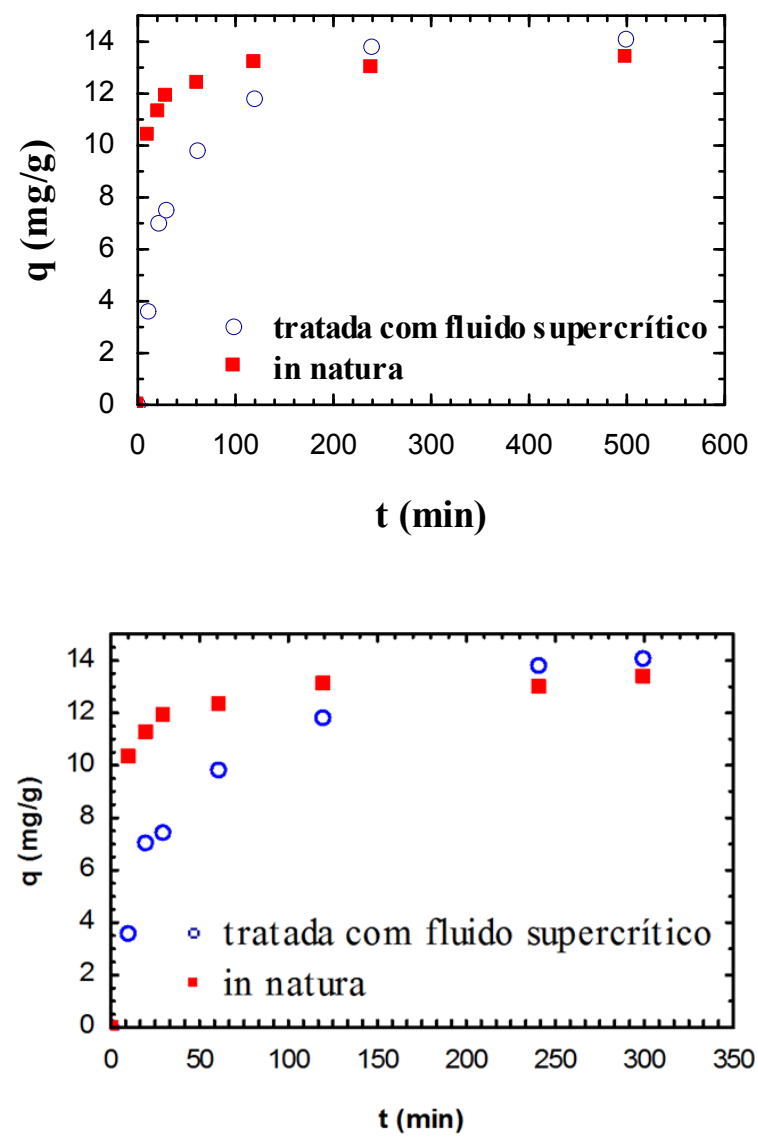

Tabela 1 - Modelos cinéticos.

\begin{tabular}{ccc}
\hline \multicolumn{3}{c}{ Pseudo-primeira ordem } \\
\hline & in natura & Tratada \\
\cline { 2 - 3 }$q_{1}\left(\mathrm{mg} \mathrm{g}^{-1}\right)$ & 12,90 & 13,33
\end{tabular}




\begin{tabular}{|c|c|c|}
\hline$k_{1}\left(\min ^{-1}\right)$ & 0,1450 & 0,0286 \\
\hline $\mathrm{R}^{2}$ & 0,8662 & 0,9716 \\
\hline EMR (\%) & 14,84 & 8,18 \\
\hline \multicolumn{3}{|c|}{ Pseudo-segunda ordem } \\
\hline & in natura & Tratada \\
\hline$q_{2}\left(\mathrm{mg} \mathrm{g}^{-1}\right)$ & 13,29 & 15,25 \\
\hline$k_{2}\left(\mathrm{~g} \mathrm{mg}^{-1} \mathrm{~min}^{-1}\right)$ & 0,0054 & 0,0022 \\
\hline $\mathrm{R}^{2}$ & 0,9801 & 0,9929 \\
\hline $\operatorname{EMR}(\%)$ & 1,63 & 6,65 \\
\hline
\end{tabular}

A capacidade de adsorção da casca de arroz in natura atingiu o equilíbrio em cerca de 120 minutos, enquanto o material tratado levou cerca de 240 minutos. A diferença nos intervalos de tempo deve-se à área superficial do material. A casca de arroz tratada possui maior porosidade e área superficial, levando a um tempo de saturação maior. Os parâmetros obtidos no ajuste estão representados na Tabela 1. A Verificou-se que, tanto para a casca in natura, quanto para a casca tratada, o modelo de pseudo-segunda ordem foi o mais adequado para representar a cinética de adsorção, apresentando $\mathrm{R}^{2}$ mais próximos da unidade e menores valores de erro médio relativo (EMR). Os valores de $q_{2}$ foram maiores para a casca tratada, confirmando que acasca de arroz tratada atingiu uma maior capacidade de adsorção no equilíbrio.

\subsection{Estudo do equilíbrio}

Os dados experimentais de equilíbrio estão representados na Figura4, onde se visualiza que a capacidade máxima de adsorção experimental passou de $18 \mathrm{mgg}^{-1}$ para $22 \mathrm{mg} \mathrm{g}^{-1}$ com o tratamento via $\mathrm{CO}_{2}$ supercrítico.

Figura 4 - Curva do equilíbrio de adsorção.

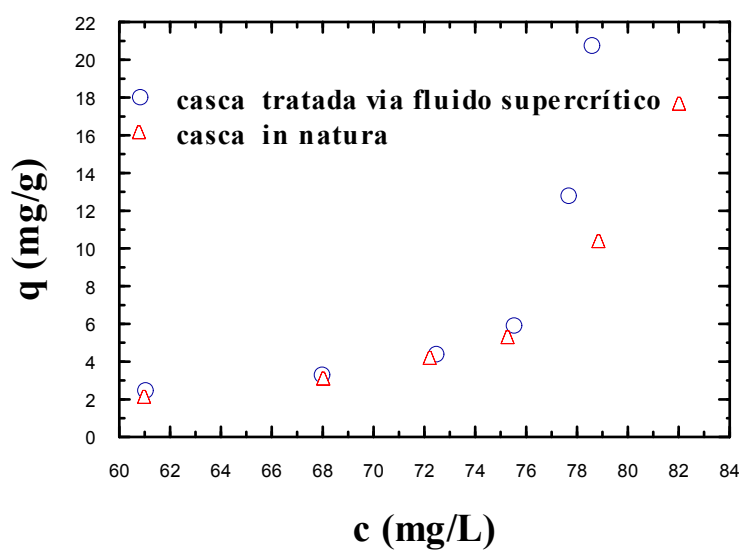

Tabela 2 - Modelos de equilíbrio. 


\begin{tabular}{ccc}
\hline & in natura & tratada \\
\cline { 2 - 3 }$k_{F}$ & 0,000022 & 0,000098 \\
$1 / n_{\mathrm{f}}$ & 0,149 & 0,151 \\
$\mathrm{R}^{2}$ & 0,9866 & 0,8797 \\
EMR $(\%)$ & 10,47 & 24,82 \\
\hline & BET & tratada \\
\cline { 2 - 3 }$q_{B E T}\left(\mathrm{mg} \mathrm{g}^{-1}\right)$ & 13,0 & 28,82 \\
$K_{l}\left(\mathrm{~L} \mathrm{mg}^{-1}\right)$ & 0,000325 & 0,00083 \\
$K_{2}\left(\mathrm{~L} \mathrm{mg}^{-1}\right)$ & 0,0110 & 0,01165 \\
$\mathrm{R}^{2}$ & 0,9828 & 0,9835 \\
EMR (\%) & 9,97 & 9,15 \\
\hline
\end{tabular}

Na Tabela 2 encontram-se os parâmetros obtidos no ajuste das isotermas na temperatura de $55^{\circ} \mathrm{C}$. O modelo de BET foi o mais adequado para representar o comportamento dos dados experimentais para os dois materiais (EMR $\left.<10 \%, \mathrm{R}^{2}>0,98\right)$, e a capacidade estimada por ele foi de $13 \mathrm{mg} \mathrm{g}^{-1}$ para a casca de arroz sem tratamento e $28,82 \mathrm{mg} \mathrm{g}^{-1}$ para a mesma tratada com fluido supercrítico, indicando um aumento de $121 \%$, favorável ao uso do tratamento.

Em geral, acapacidade da casca de arroz em recuperar cobalto aumentou com o tratamento supercrítico em todos os estudos realizados. Esse efeito pode ser explicado pelas alterações estruturais no material e aumento da área superficial, os quais foram causados pela alta pressão e interações com o solvente.

\section{CONCLUSÃO}

Tendo em vista os resultados alcançados neste trabalho, conclui-se que o tratamento utilizando $\mathrm{CO}_{2}$ supercrítico é benéfico para o material adsorvente utilizado, aumentando a capacidade da casca de arroz de remover cobalto de soluções aquosas.Pode-se observar, através dos parâmetros de regressão encontrados nas Tabelas 1 e 2, que o comportamento cinético adaptou-se melhor ao modelo de pseudo-segunda ordem, enquanto que o modelo BET mostrou-se o mais adequado para os dados do equilíbrio. A máxima capacidade de adsorção aumentou $121 \%$ para o material tratado em relação ao sem tratamento.

\section{REFERÊNCIAS}

ORLANDO, U. S. et al. Preparation of agricultural residue anion exchangers and its nitrate maximum adsorption capacity.Chemosphere, v. 48, 2002.

SAFA, Y., BHATTI, H.N. Biosorption of Direct Red-31 and Direct Orange-26 dyes by rice husk: Application of factorial design analysis. Chemical Engineering Research and Design, v. 798, 2011.

SANTOS, J. C. dos.Extração com fluido supercrítico e suas aplicações na obtenção de produtos naturais.2011. 40 f. Trabalho de conclusão de curso(Graduação em Farmácia)Universidade Federal do Rio Grande do Sul, Porto Alegre, 2011. 
SULAYMON, A. H. et al. Removal of lead copper chromium and cobalt ions onto granular activated carbon in batch and fixed-bed adsorbers.Chemical Engineering Journal, v. 155, p. 647, 2009.

VIEIRA, M. G. A. et al. Characterization and use of in natura and calcined rice husks for biosorption of heavy metals ions from aqueous effluents. Brazilian Journal of Chemical Engineering, v. 29, p. 619, 2012. 